\title{
The Adrenal Gland as a Sanctuary Site of Metastases After Pembrolizumab Treatment: A Case Series
}

\author{
Michelle C. Nguyen, MD, MPH ${ }^{\mathrm{a}}$; Manisha H. Shah, $\mathrm{MD}^{\mathrm{b}}$; David A. Liebner, MD ${ }^{\mathrm{b}}$; Floor J. Backes, $\mathrm{MD}^{\mathrm{b}}$; \\ John Phay, $\mathrm{MD}^{\mathrm{b}}$; and Lawrence A. Shirley, $\mathrm{MD}^{\mathrm{b}}$
}

\begin{abstract}
Therapeutic agents targeting the PD-1/PD-L1 axis have shown durable clinical responses in patients with various cancer types. Although objective responses are common, intrapatient heterogeneous responses have been described, and the mechanism for the different organ responses remains unknown. We present a series of patients in whom a lack of response was noted solely in the adrenal glands. This is the first case series describing 3 patients with heterogeneous patterns of response to pembrolizumab with progression of adrenal metastatic disease despite objective response (complete or partial response) in all other sites of metastatic disease. Two patients, one with melanoma and one with uterine carcinosarcoma, underwent robotic adrenalectomy for enlarging adrenal metastases. An additional patient with melanoma underwent laparotomy with attempted resection, but infiltration of the adrenal tumor into the inferior vena cava prohibited safe excision. This report provides additional insight into the heterogeneous patterns of disease response to anti-PD-1 therapy, highlighting the adrenal gland as a potential sanctuary site for this immunotherapy. These cases display the potential benefit of early surgical resection in this scenario and the pitfalls of delaying referral to a surgeon for assessment of operative intervention.
\end{abstract}

J Natl Compr Canc Netw 2018;16(11):1279-1283 doi: 10.6004/jnccn.2018.7059

\section{Background}

The development of therapeutic agents targeting the PD-1/PD-L1 axis has been a major therapeutic advancement in immuno-oncology. ${ }^{1}$ Pembrolizumab is the first anti-PD-1 antibody to be FDA approved for the treatment of patients with unresectable or metastatic melanoma who have experienced progression after treatment with ipilimumab and, if BRAF V600-mutation positive, a BRAF inhibitor. ${ }^{2}$ Since then, pembrolizumab has also received FDA approval for the treatment of advanced non-small cell lung cancer, classical Hodgkin lymphoma, gastric cancer, head and neck squamous cell cancer, urothelial bladder cancer, and microsatellite-unstable

\footnotetext{
aThe Ohio State University Wexner Medical Center, and ${ }^{\mathrm{b}}$ The Ohio State University James Comprehensive Cancer Center, Columbus, Ohio.

Submitted March 25, 2018; accepted for publication July 9, 2018.

Dr. Shah has disclosed that she receives grant/research support from Eisai, Loxo Oncology, and Merck; and that she serves on the advisory board for Eisai, Loxo Oncology, Ignyta, and Novartis. Dr. Backes has disclosed
}

tumors. Multiple clinical trials are currently underway to evaluate its effectiveness in other solid tumor types, including advanced triple-negative breast cancer and locally advanced or metastatic endometrial cancer. ${ }^{3-7}$

In advanced-stage melanoma, anti-PD-1 therapy has shown marked clinical activity, with response rates of approximately $40 \%$ and a reduction in overall tumor burden in $60 \%$ to $70 \%$ of patients. However, there are still $30 \%$ to $40 \%$ of patients in whom the response is heterogeneous or no tumor regression is seen. ${ }^{8-10}$ Variable metastasis-specific response to anti-PD-1 has also been shown for melanoma metastases to the lung, liver, subcutaneous and peritoneal tissue, and lymph nodes. The objective response appears to be highest for smaller

that she serves on the advisory board and is a consultant for Merck. The remaining authors have disclosed that they have no financial interests, arrangements, affiliations, or commercial interests with the manufacturers of any products discussed in this article or their competitors.

Correspondence: Michelle C. Nguyen, MD, MPH, The Ohio State University Wexner Medical Center, 395 West 12th Avenue, Columbus, OH 43210. Email: michelle.nguyen@osumc.edu 
Nguyen et al

metastatic lesions and those to the lung, with the most variable responses seen in the liver. ${ }^{11-13}$ The differences in metastatic-specific patterns of response suggest differences in tumor microenvironment that may have implications in patient management. Currently, there are no reports of response rates for metastatic adrenal masses in patients receiving antiPD-1 therapy. This is the first case series describing 3 patients with heterogeneous patterns of response to pembrolizumab with progression of adrenal metastatic disease despite objective response in other sites of distant metastatic disease.

\section{Case Presentation}

Case 1

A 64-year-old woman with cutaneous melanoma originally treated with wide local excision in 2008, later developed metastatic disease to the brain, lung, retroperitoneum, and cervical nodes in 2013. She underwent a craniotomy and resection of a hemorrhagic brain lesion, with pathology results consistent with a 2.8-cm deposit of metastatic melanoma. Whole-brain radiation therapy (RT) with 30 Gy and 5 cycles of ipilimumab were completed, and restaging scans demonstrated stable metastatic disease. Three months later, the patient was found to have interval progression of retroperitoneal disease and was started on maintenance ipilimumab. Progressive brain, cervical, lung, and abdominal disease was identified shortly thereafter, and she was started on trametinib but stopped after 1 cycle due to adverse reactions. A third-line therapy with pembrolizumab at $2 \mathrm{mg} / \mathrm{kg}$ administered intravenously every 21 days was initiated, resulting in significant response. After 30 cycles of pembrolizumab over a 2-year period, the patient was found to have a new and enlarging right adrenal gland mass measuring $1.9 \times 3.1 \mathrm{~cm}$ (Figure 1) despite stability of brain lesions, and retroperitoneal and cervical nodes. PET imaging confirmed a hypermetabolic right adrenal mass. The patient underwent right robotic adrenalectomy in 2016, and pathology results confirmed metastatic melanoma. Pembrolizumab was continued during this time. One year later, the patient developed progression of brain metastases and underwent stereotactic radiosurgery 4 times. Pembrolizumab was eventually held in
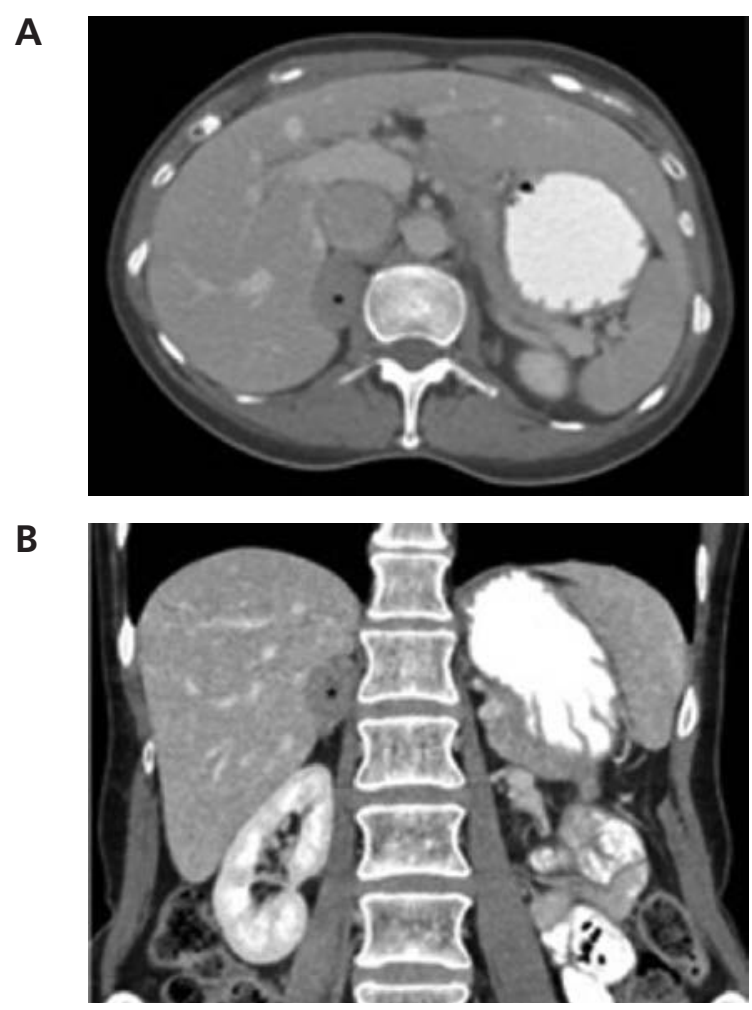

Figure 1. (A) Axial and (B) coronal CT images of the abdomen demonstrating a right adrenal gland mass measuring $1.9 \times 3.1 \mathrm{~cm}$.

January 2018 due to central nervous system disease progression.

\section{Case 2}

A 71-year-old woman underwent wide excision of a left forearm melanoma in 2009. Seven years later, she developed left shoulder discomfort for which imaging demonstrated a large heterogeneous mass with necrosis involving the scapula and invading surrounding musculature, in addition to left axillary lymphadenopathy, right pulmonary nodules, and an indeterminate 1.4$\mathrm{cm}$ hyperdense right perirenal lesion. Biopsy results of the left scapular and left axillary nodes and right pulmonary nodule revealed metastatic melanoma. RT to the scapula was completed, and the patient was started on pembrolizumab at $2 \mathrm{mg} / \mathrm{kg}$ intravenously every 21 days. Within 3 months of treatment initiation, a significant response to scapular, axillary, and pulmonary lesions was seen. Eight months later, the patient developed right upper quadrant abdominal pain and was found on CT imaging to have an $8.3 \times 7.8-\mathrm{cm}$ mass arising from the right adrenal gland. Restaging CT scan of the chest during that time showed an interval decrease in size of pulmonary nodules as well as 
a decrease in size of the left scapular lesion consistent with positive interval treatment response. Biopsy results of the right adrenal lesion were consistent with metastatic melanoma. A PET scan performed shortly thereafter showed an increase in size of the right adrenal mass to $9.6 \times 8.8 \mathrm{~cm}$ with no significant uptake in the left shoulder, axilla, or lung. Two months later, the patient was sent for surgical evaluation and a preoperative CT scan showed rapid growth of the right adrenal lesion to $10 \times 11.4 \times 12.9 \mathrm{~cm}$ (Figure 2). The patient subsequently underwent exploratory laparotomy with attempted excision of the right adrenal mass. However, the mass was densely adherent to the right hepatic lobe, right kidney, and involved a long segment of inferior vena cava, prohibiting safe excision. She was evaluated by a radiation oncologist and completed palliative RT to the right adrenal mass at $25 \mathrm{~Gy}$ in 5 fractions in March 21, 2018. A repeat CT scan of the abdomen and pelvis 2 months later showed slight interval decrease in size of the right adrenal mass with no evidence of progression or disease elsewhere. The patient has been following up with her local oncologist for continuation of systemic therapy.

A

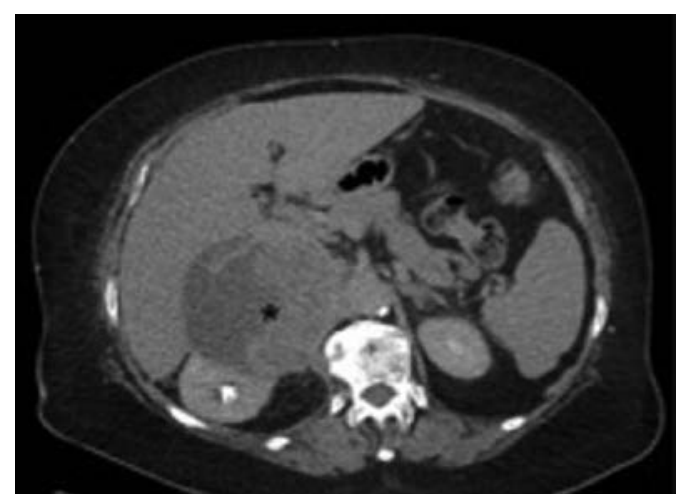

B

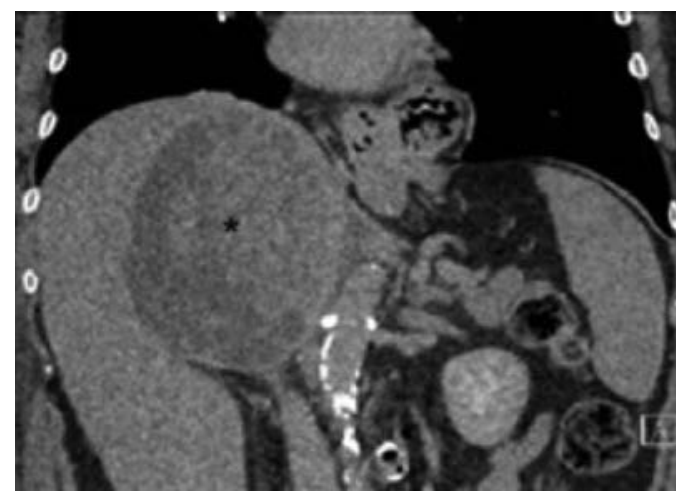

Figure 2. (A) Axial and (B) coronal CT images of the abdomen demonstrating a right adrenal lesion measuring $10.0 \times 11.4 \times 12.9 \mathrm{~cm}$.

\section{Case 3}

A 59-year-old woman underwent robotic hysterectomy with bilateral salpingo-oophorectomy and pelvic and periaortic lymph node dissection for stage III uterine carcinosarcoma in March 2016. She completed 6 cycles of carboplatin and paclitaxel therapy and 4,860 cGy of RT to the pelvic and aortic nodes. She later developed subcutaneous nodules and breast lesions with biopsy results confirming poorly differentiated carcinoma compatible with a metastasis of uterine origin. Staging at that time also showed multiple subcutaneous nodules and peritoneal and hilar lesions. The patient was started on pembrolizumab at $200 \mathrm{mg}$ intravenously every 21 days, resulting in a significant interval decrease in size of the lesions. Restaging scans 1 month later demonstrated a new right adrenal mass measuring $1.4 \times 2.4 \mathrm{~cm}$ and left adrenal nodule measuring $1.9 \times 2 \mathrm{~cm}$. After an additional 3 months of pembrolizumab therapy, restaging scans showed an increase in bilateral adrenal masses to $2.3 \times 4.3 \mathrm{~cm}$ on the left and $3.3 \times 3.3 \mathrm{~cm}$ on the right (Figure 3 ), despite resolution of subcutaneous and retroperitoneal nodes and stability of hilar nodes. Biopsy of the left adrenal mass confirmed poorly differentiated carcinoma in a background of extensive necrosis consistent with carcinosarcoma. The patient subsequently underwent bilateral robotic adrenalectomy in February 2018, with pathology results demonstrating metastatic uterine carcinosarcoma.

\section{Discussion}

The patients presented in this case series received pembrolizumab therapy for metastatic melanoma or uterine carcinosarcoma with several sites of disease. Despite objective response to all other sites, each patient experienced progression of adrenal metastases while receiving pembrolizumab. Two patients underwent treatment with adrenalectomy without postoperative complications. This report provides additional insight into the heterogeneous patterns of response, with possible adrenal resistance to antiPD-1 therapy.

The prevalence of homogenous and heterogeneous patterns of response to anti-PD-1 agents currently remains unknown. A recent study by Lee et $\mathrm{al}^{11}$ evaluated patterns of response in 27 patients with metastatic melanoma enrolled in KEYNOTE-001. 
A

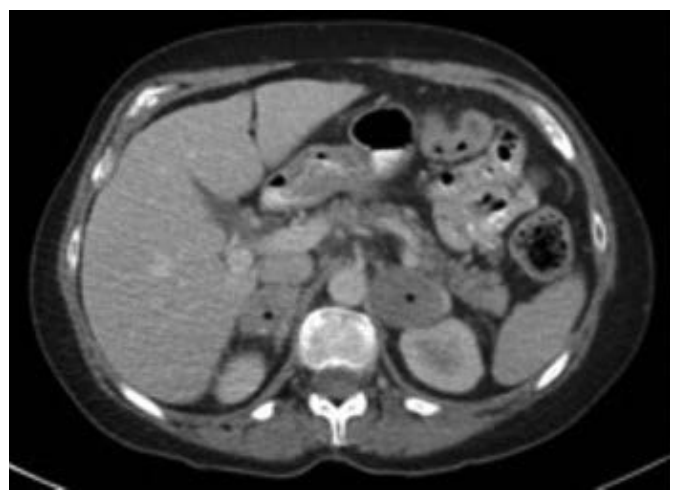

B

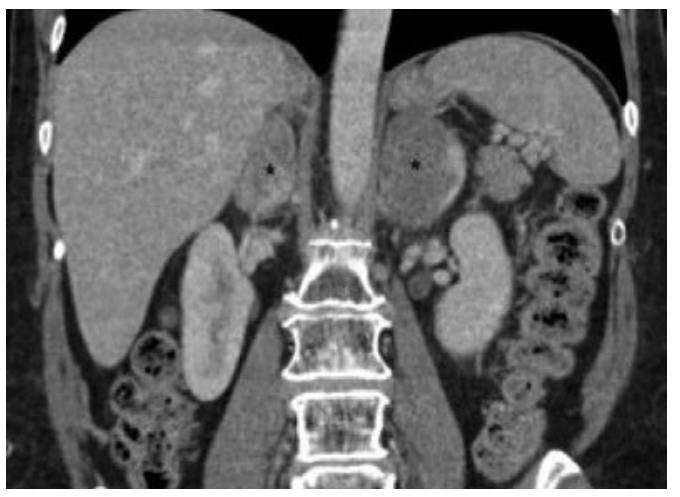

Figure 3. (A) Axial and (B) coronal CT images of the abdomen demonstrating bilateral adrenal masses measuring $2.3 \times 4.3 \mathrm{~cm}$ on the left and $3.3 \times 3.3 \mathrm{~cm}$ on the right.

Among 399 metastatic lesions, complete response to pembrolizumab was shown in $52.6 \%$ of smaller lesions (mean, 223 vs $760 \mathrm{~mm}^{2}$ ) and occurred more frequently in lung lesions. Heterogeneous responses, defined as new or progressing metastases in conjunction with complete response metastases, were seen in 4 patients with an objective response (complete or partial response). Among patients who did not achieve an objective response, $85 \%$ initially had a heterogeneous response at the first imaging assessment. With these findings, the authors suggested that early heterogeneity was an indication that an objective response to treatment would be less likely. In another report of 112 patients enrolled in KEYNOTE-101, liver metastases that were associated with treatment failure were shown to have lower CD8-positive T-cell tumor infiltration and T-cell PD-1 and tumor PD-L1 expression. Lung lesions were associated with higher rates of objective response, consistent with findings from previous studies. ${ }^{13}$ The differential responses seen in the liver compared with the consistent responses in the lung suggests that differences in tumor microenvironments may play a sig- nificant role in the heterogeneity of responses. This hypothesis has also been supported by Herbst et al, ${ }^{14}$ who demonstrated associations in tumor expression of PD-L1, T-helper type 1 gene, and CTLA4, and absence of fractalkine (CX3CL1) in baseline tumors with response rates.

The tumor microenvironment of metastatic adrenal masses and its associated response to anti-PD-1 therapy is currently unknown. However, it is well-established that these immune checkpoint inhibitors (ICIs) are associated with immune-related endocrinopathies, including adrenal insufficiency. ${ }^{15-18}$ In a meta-analysis of 10 clinical trials representative of 3,278 patients receiving ICI therapy, adrenal insufficiency was reported in $0.8 \%$ to $2 \%$ of those with a relative risk of $3.87 .{ }^{19}$ The exact mechanism remains unclear but perhaps may play a role in the heterogeneous responses of adrenal metastases to anti-PD-1 therapy.

The existence of areas within the body, particularly the brain and testis, that are relatively protected from the cytotoxic effects of chemotherapy has led to the recognition of "sanctuary sites." Sanctuary sites have been particularly associated with hematologic malignancies, ${ }^{20}$ but evidence for the brain as a sanctuary site in small cell lung $^{21}$ and ovarian cancers ${ }^{22}$ has been described. The testis is also a site protected from chemotherapy that is well-documented in leukemia treatment and reported in testicular cancer. ${ }^{23}$ Currently, no reports have suggested the adrenal gland as a potential sanctuary site for certain malignancies, such as melanoma or uterine cancer.

Furthermore, the sensitivity of adrenal metastases to other systemic or targeted therapies across other malignancies remains to be elucidated, because patients with cancer diagnosed with clinically isolated adrenal metastases are often referred for surgical treatment. Studies have shown that resection of clinically isolated adrenal metastases results in increased median and overall survival compared with nonsurgical therapy, and in fact earlier intervention in selected patients may provide survival benefits. ${ }^{24-26}$ However, the benefit of adrenalectomy over systemic therapy in patients with poor prognoses is questionable. In a study of patients who underwent resection of colorectal metastases, adrenal metastasis was associated with a poor prognosis and adrenalectomy did not offer any benefit over chemotherapy alone. ${ }^{27}$ For other malignancies, such as small bowel 
adenocarcinomas, chemotherapy alone may be sufficient. In one case report, a patient who underwent surgical resection of a duodenal carcinoma was later diagnosed with bilateral adrenal metastases and was successfully treated with 6 cycles of docetaxel/ cisplatin/5-fluorouracil chemotherapy. ${ }^{28}$ These data suggest the need for continued investigation of adrenal metastases, their tumor microenvironment, and their immune cell profile to better understand their response to different systemic or targeted therapies, especially if local resection is contraindicated.

Although our report is limited by the small series of only 3 patients, our observations provide initial insight to guide future studies investigating the resistance of adrenal metastases to immunotherapy. Our group is planning future studies to explore the microenvironment of tumor deposits responsive to immunotherapy versus the microenvironment of persistent adrenal metastases.

\section{Conclusions}

This report provides additional insight to the heterogeneous patterns of response to anti-PD-1 therapy, which may have implications for patient management. The progression of adrenal metastatic lesion with stability or improvement of other sites of disease implicates the importance of early operative intervention for treatment. Larger series of adrenal metastases and response to anti-PD-1 will further elucidate our initial observations.

\section{References}

1. Couzin-Frankel J. Breakthrough of the year 2013. Cancer immunotherapy. Science 2013;342:1432-1433.

2. Chuk MK, Chang JT, Theoret MR, et al. FDA approval summary: accelerated approval of pembrolizumab for second-line treatment of metastatic melanoma. Clin Cancer Res 2017;23:5666-5670.

3. Muro K, Chung HC, Shankaran V, et al. Pembrolizumab for patients with PD-L1-positive advanced gastric cancer (KEYNOTE-012): a multicentre, open-label, phase $1 \mathrm{~b}$ trial. Lancet Oncol 2016;17:717-726.

4. Plimack ER, Bellmunt J, Gupta S, et al. Safety and activity of pembrolizumab in patients with locally advanced or metastatic urothelial cancer (KEYNOTE-012): a non-randomised, open-label, phase $1 \mathrm{~b}$ study. Lancet Oncol 2017;18:212-220.

5. Seiwert TY, Burtness B, Mehra R, et al. Safety and clinical activity of pembrolizumab for treatment of recurrent or metastatic squamous cell carcinoma of the head and neck (KEYNOTE-012): an open-label, multicentre, phase 1b trial. Lancet Oncol 2016;17:956-965.

6. Nanda R, Chow LQ, Dees EC, et al. Pembrolizumab in patients with advanced triple-negative breast cancer: phase Ib KEYNOTE-012 study. J Clin Oncol 2016;34:2460-2467.

7. Ott PA, Bang YJ, Berton-Rigaud D, et al. Safety and antitumor activity of pembrolizumab in advanced programmed death ligand 1-positive endometrial cancer: results from the KEYNOTE-028 study. J Clin Oncol 2017;35:2535-2541.

8. Larkin J, Hodi FS, Wolchok JD. Combined nivolumab and ipilimumab or monotherapy in untreated melanoma. N Engl J Med 2015;373:1270-1271.

9. Ribas A, Hamid O, Daud A, et al. Association of pembrolizumab with tumor response and survival among patients with advanced melanoma. JAMA 2016;315:1600-1609.

10. Robert C, Schachter J, Long GV, et al. Pembrolizumab versus ipilimumab in advanced melanoma. N Engl J Med 2015;372:2521-2532.

11. Lee JH, Lyle M, Menzies AM, et al. Metastasis-specific patterns of response and progression with anti-PD-1 treatment in metastatic melanoma. Pigment Cell Melanoma Res 2018;31:404-410.

12. Khoja L, Kibrio M, Metser U, et al. Patterns of response to anti-PD-1 treatment: an exploratory comparison of four radiological response criteria and associations with overall survival in metastatic melanoma patients. $\mathrm{Br}$ J Cancer 2016;115:1186-1192.

13. Tumeh PC, Harview CL, Yearley JH, et al. PD-1 blockade induces responses by inhibiting adaptive immune resistance. Nature 2014;515:568-571.

14. Herbst RS, Soria JC, Kowanetz M, et al. Predictive correlates of response to the anti-PD-L1 antibody MPDL3280A in cancer patients. Nature 2014;515:563-567.

15. Villa NM, Farahmand A, Du L, et al. Endocrinopathies with use of cancer immunotherapies. Clin Endocrinol (Oxf) 2018;88:327-332.

16. Sznol M, Postow MA, Davies MJ, et al. Endocrine-related adverse events associated with immune checkpoint blockade and expert insights on their management. Cancer Treat Rev 2017;58:70-76.

17. Cukier P, Santini FC. Scaranti M, Hoff AO. Endocrine side effects of cancer immunotherapy. Endocr Relat Cancer 2017;24:T331-347.

18. Ariyasu R, Horiike A, Yoshizama $T$, et al. Adrenal insufficiency related to anti-programmed death-1 therapy. Anticancer Res 2017;37:4229-4232.

19. Abdel-Rahman O, ElHalawani H, Fouad M. Risk of endocrine complications in cancer patients treated with immune check point inhibitors: a meta-analysis. Future Oncol 2016;12:413-425.

20. Larson RA. Managing CNS disease in adults with acute lymphoblastic leukemia. Leuk Lymphoma 2018;59:3-13.

21. Glantz MJ, Choy H, Yee L. Prophylactic cranial irradiation in small cell lung cancer: rationale, results, and recommendations. Semin Oncol 1997;24:477-483.

22. Cormio G, Maneo A, Parma G, et al. Central nervous system metastases in patients with ovarian carcinoma. A report of 23 cases and a literature review. Ann Oncol 1995;6:571-574.

23. Palmieri D, Chambers AF, Felding-Habermann B, et al. The biology of metastasis to a sanctuary site. Clin Cancer Res 2007;13:1656-1662.

24. Sarela AI, Murphy I, Coit DG, Conlon KC. Metastasis to the adrenal gland: the emerging role of laparoscopic surgery. Ann Surg Oncol 2003;10:1191-1196.

25. Duh QY. Resecting isolated adrenal metastasis: why and how? Ann Surg Oncol 2003;10:1138-1139.

26. Strong VE, D'Angelica M, Tang L, et al. Laparoscopic adrenalectomy for isolated adrenal metastasis. Ann Surg Oncol 2007;14:3392-3400.

27. de Haas RJ, Rahy Martin AC, Wicherts DA, et al. Long-term outcome in patients with adrenal metastases following resection of colorectal liver metastases. Br J Surg 2009;96:935-940.

28. Bitik B, Kalpakci Y, Altan E, et al. Successful treatment of primary duodenal carcinoma with bilateral adrenal metastases with docetaxelcisplatin-5-fluorouracil regimen. Ann Oncol 2009;20:394-395. 